\title{
Modeling the gas reservoir of circumstellar disks around young G-type stars
}

\author{
I. Kamp ${ }^{1,2}$ and F. Sammar ${ }^{1}$ \\ ${ }^{1}$ Leiden Observatory, PO Box 9513, 2300 RA Leiden, The Netherlands \\ 2 Space Telescope Science Institute, 3700 San Martin Drive, Baltimore, MD 21218, USA \\ e-mail: kamp@stsci .edu
}

Received 27 August 2003 / Accepted 27 July 2004

\begin{abstract}
Interpretation of molecular line observations in tenuous circumstellar disks around young G-type stars in terms of a disk mass is difficult without a model that describes the chemical structure of these disks. This paper discusses the chemistry in tenuous disks around young solar-type stars based on disk models that take into account the presence of a stellar chromosphere. The example of the disk around a $70 \mathrm{Myr}$ old solar-type star shows that the dissociating radiation from the chromosphere is stronger than the interstellar ultraviolet radiation field up to a distance of $\sim 400 \mathrm{AU}$ from the star. Similar to other studies in this research field, it is found that, due to photodissociation, the CO-to- $\mathrm{H}_{2}$ ratio is far from the canonical value of $10^{-4}$ for molecular clouds. Moreover, the dust-to-gas mass ratio as well as the dust grain size plays an important role for the $\mathrm{H}_{2}$ abundance in these disks.
\end{abstract}

Key words. stars: chromospheres - stars: circumstellar matter - stars: pre-main sequence - stars: individual: HD 123160

\section{Introduction}

The IRAS and ISO satellites revealed that a large fraction of nearby pre-main sequence solar-type stars are surrounded by cool dust. Near-infrared and submillimeter observations have shown that the dust is distributed in disk-like, sometimes ringlike structures around the star (see Zuckerman 2001 for a recent review). However, the gas content of these protoplanetary disks is still under debate. $\mathrm{CO}$ observations by Zuckerman et al. (1995) for a sample of young stars in the range $10^{6}-10^{7} \mathrm{yr}$ lead to the conclusion that the gas dissipates very rapidly within a few million years - from the protoplanetary disks. A later study by Coulson et al. (1998) for a sample of 24 candidate Vega-excess stars also shows too little or no $\mathrm{CO}$ emission. Only recently, Greaves et al. (2000) did not find any evidence for $\mathrm{CO}$ rotational lines in a sample of 14 nearby $\mathrm{F}$ and G-type stars with known circumstellar dust. Since solar-type stars are thought to have little UV radiation, the non-detection of $\mathrm{CO}$ is generally interpreted as an overall lack of gas in the disks around these stars. As a consequence planets would have to form either faster in the framework of the core-accretion model or on a very short timescale - a few thousand years - via gravitational instabilities.

What people have neglected so far is the possible existence of a chromosphere in solar-type stars. Ayres (1997) has shown that the ionizing ultraviolet flux from our Sun was much stronger in the past and evolved roughly as $t^{-1}$ with time. The aim of this paper is to study the influence of a chromosphere on the chemistry in late phases of disk evolution, that is for disks with $L_{\mathrm{IR}} / L_{*}<0.01$. For this purpose, we choose a template for a $70 \mathrm{Myr}$ old solar-type star and compile a typical chromospheric radiation field (Sect. 2). The modeling procedure is briefly described in Sect. 3 and the outcome of the model calculations is presented and discussed in Sect. 4.

\section{The template G-type star}

The initial search for a template star was carried out with the following criteria:

- The spectral type has to be similar to our Sun, G5v.

- $L_{\mathrm{IR}} / L_{*}<0.01$, that is the disk has to be optically thin in the UV.

- Disk parameters have to be known from infrared photometry.

- CO observations have to exist (either detection or upper limit).

From a literature study of the papers by Sylvester \& Skinner (1996), Sylvester et al. (1996), Dunkin et al. (1997a,b), Zuckerman et al. (1995), Coulson et al. (1998) and Greaves et al. (2000), only one candidate star was found: HD 123160. Unfortunately, the spectral type as well as the nature of the infrared excess of this star was recently questioned: Mora et al. (2001) and Kalas et al. (2002) classify this star as a K5 giant and detect a complex reflection nebula instead of a disk-like structure. We conclude that observational data are simply too 
Table 1. Stellar parameters and disk parameters for the template star. The disk parameters in brackets refer to an alternative disk model that would result in a very similar IR excess.

\begin{tabular}{ll}
\hline \hline Parameter & Template star \\
\hline Spectral type & G5 V \\
$T_{\text {eff }}$ & 5570 \\
$\log g$ & 4.5 \\
Stellar radius & $0.9 R_{\odot}$ \\
Distance & $15.7 \mathrm{pc}$ \\
Age & $70 \times 10^{6} \mathrm{yr}$ \\
$R_{\mathrm{i}}($ disk $)$ & $190 \mathrm{AU}$ \\
& $(60 \mathrm{AU})$ \\
$R_{\mathrm{o}}($ disk $)$ & $470 \mathrm{AU}$ \\
$L_{\mathrm{IR}} / L_{*}$ & $4.4 \times 10^{-3}$ \\
$M_{\text {dust }}($ disk $)$ & $0.057 M_{\oplus}$ \\
& $\left(0.77 M_{\oplus}\right)$ \\
\hline
\end{tabular}

scarce up to now, and we proceed by compiling a template star using typical stellar and disk properties.

\subsection{Stellar parameters and disk properties}

The basic stellar parameters and disk parameters assumed for the template star are summarized in Table 1. We assume in the following a typical age of $70 \mathrm{Myr}$ for our template.

As in most cases observations only yield a lower limit for the gas mass, the disk models are calculated for three different gas masses: $0.033,0.33$ and $3.3 M_{\oplus}$. On the other hand, the dust mass is fixed to $0.057 M_{\oplus}$. This means that the gas-todust mass ratio varies in the three models from 0.58 to 58 . In addition, model calculations are performed with an alternative more massive dust disk model, $M_{\text {dust }}=0.77 M_{\oplus}$, which shows a very similar IR excess.

\subsection{The UV radiation field}

Following Table 1, a Kurucz ATLAS9 stellar atmosphere model (Kurucz 1992) with an effective temperature of $5750 \mathrm{~K}$ and a $\log g$ of 4.5 is assumed for our template star.

In early stages of star formation, the ultraviolet radiation field is dominated by active accretion from the circumstellar disk/envelope. In later stages, when accretion is no longer the dominant process, stellar activity takes over. Studies of open star clusters have shown that young solar-type stars are more active than the present Sun. Ayres (1997) estimated correlations between stellar activity and rotation for different tracers like O VI, C III and Ly $\alpha$ based on observed X-ray-rotation and C IVrotation relations. Combining this with observed age-rotation relations, Ayres concludes that all the photorates scale approximately as $\sim t^{-1}$ with time. Photodissociation of $\mathrm{CO}$ and $\mathrm{H}_{2}$ takes place through discrete bands and is thus affected mostly by the continuum. Nevertheless, some of the dissociating bands overlap with the emission lines of an active solar-type star, e.g. with the O VI emission around $1038 \AA$ or C III at $977 \AA$. Hence, it is assumed that the total flux in the 912-1110 $\AA$ region scales with time as $\sim t^{-1}$.

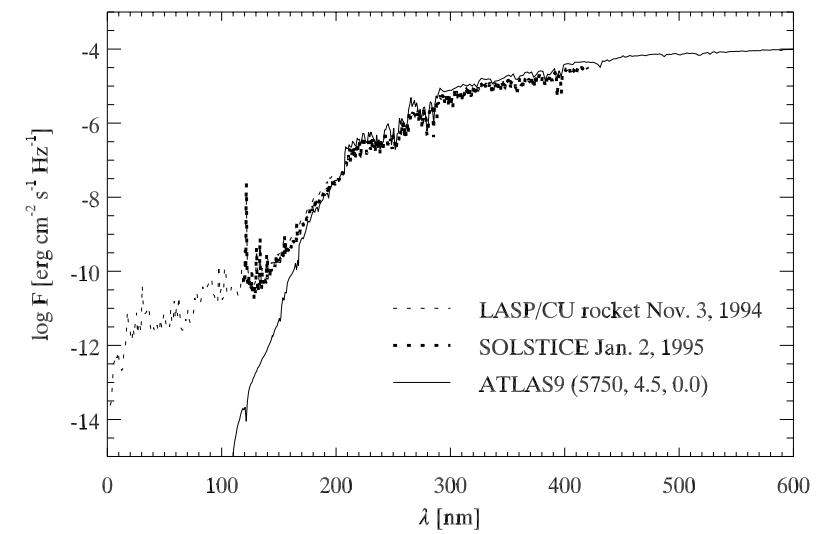

Fig. 1. Flux distribution of the Sun at the solar surface. Solid line: Kurucz ATLAS9 model with $T_{\text {eff }}=5750 \mathrm{~K}$ and $\log g=4.5$. Thin dotted line: LASP/CU rocket experiment. Thick dotted line: SOLSTICE.

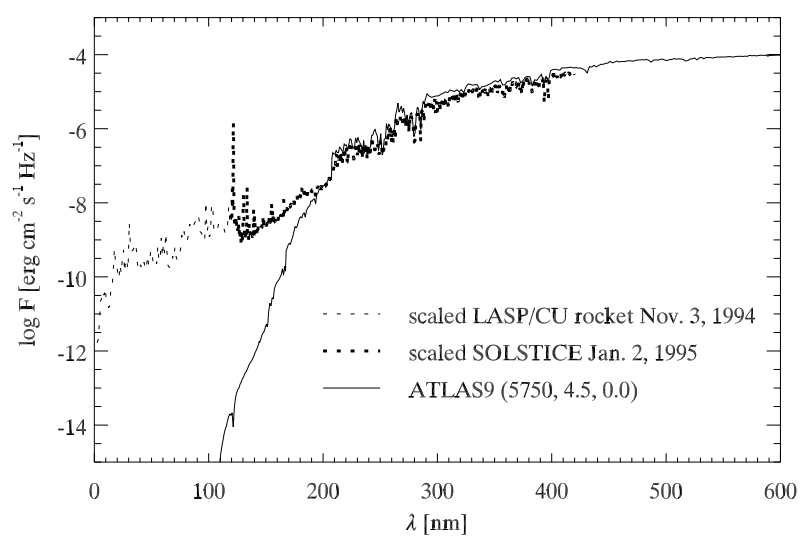

Fig. 2. Flux distribution at the stellar surface of the template star. Solid line: Kurucz ATLAS9 model with $T_{\text {eff }}=5750 \mathrm{~K}$ and $\log g=4.5$. Thin dotted line: LASP/CU rocket experiment data scaled by a factor 70 . Thick dotted line: SOLSTICE data scaled according to Eq. (1).

Data from the LASP/CU rocket experiment and the Solar Stellar Irradiance Comparison Experiment (SOLSTICE) serve as a template for the UV radiation of our present Sun. Figure 1 shows that the rocket and satellite data agree well with a Kurucz ATLAS9 model of $5750 \mathrm{~K}$ and a $\log g$ of 4.5 .

With an age of $70 \mathrm{Myr}$ for our template star, the flux has to be scaled by a factor $\alpha=70$ with regard to the Sun. Since Ayres (1997) states that the linear scaling of the flux does not hold for processes involving the continuum at wavelengths larger than $1500 \AA$, a different scaling law is used for the 912-1190 range (rocket data) and the 1190-2000 $\AA$ range (SOLSTICE data). The first wavelength interval is scaled up by a constant factor of 70. To match the photospheric flux at $2000 \AA$, the second interval is then scaled according to the following relation

$\log F_{v}($ template $)=\log F_{v}($ SOLSTICE $)$

$$
+\frac{\log \alpha}{81}(2000-\lambda)
$$

where $F_{v}$ is given in erg $\mathrm{cm}^{-2} \mathrm{~s}^{-1} \mathrm{~Hz}^{-1}$ and $\lambda$ is in $\AA$. The resulting flux distribution is depicted in Fig. 2. Our high resolution spectrum overlaps with previously published reconstructed near UV fluxes for the 70 Myr old Sun 


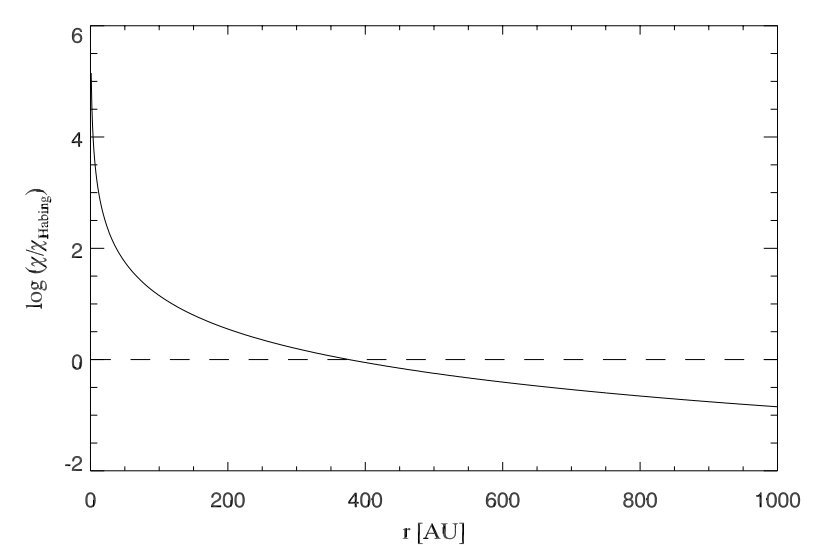

Fig. 3. Unattenuated integrated UV flux (912-1110 ̊̊) normalized to the interstellar Habing flux as a function of distance from the star; solid line: template star plus chromosphere, dashed line: Habing field.

(Dorren \& Guinan 1994). The latter does not cover the UV region of interest, where $\mathrm{CO}$ and $\mathrm{H}_{2}$ photodissociate. However, in the overlap region beyond $1250 \AA$, the overall agreement is very good.

Figure 3 illustrates the strength $\chi$ of the scaled UV radiation field with respect to an interstellar Habing field (Habing 1968), $1.222 \times 10^{7} \mathrm{~cm}^{-2} \mathrm{~s}^{-1} \cdot \chi$ denotes the integrated UV flux over the range 912-1110 $\AA$. It is clearly seen that the stellar radiation field dominates up to $\sim 400 \mathrm{AU}$ in an optically thin disk.

\section{The disk model}

The main features of the disk models are briefly summarized; for further details we refer to the original paper of Kamp \& Bertoldi (2000).

We assume thin hydrostatic equilibrium models

$n(r, z)=n_{\mathrm{i}}\left(r / R_{\mathrm{i}}\right)^{-2.5} \mathrm{e}^{-z^{2} / 2 h^{2}}$.

As a simplification, we assume a dimensionless scale-height $H \equiv h / r=0.15$. This does not lead to fully self-consistent disk models, but a coupling of the gas temperature calculation and the disk structure calculation is beyond the scope of this paper. The inner and outer radius of the disk are set to the respective values given in Table 1 . The power-law exponent of the disk surface density is -1.5 . The stellar radiation field $F_{v}$ is described above (see Sect. 2.2).

The dust temperature follows from radiative equilibrium assuming large spherical grains of radius $a=3 \mu \mathrm{m}$ (Kamp \& Bertoldi 2000)

$T_{\text {dust }}=282.5\left(L_{*} / L_{\odot}\right)^{1 / 5}(r / \mathrm{AU})^{-2 / 5}(a / \mu \mathrm{m})^{-1 / 5}$,

with the stellar luminosity in units of the solar luminosity $L_{\odot}$. The assumption of this simple form of radiative equilibrium is certainly correct for the large dust grains and optically thin disks described in this paper.

The chemical network consists of 47 atomic, ionic, and molecular species that are related through 268 gas-phase chemical and photoreactions. A number of reactions is treated in more detail like $\mathrm{H}_{2}$ and $\mathrm{CO}$ photo-dissociation, and $\mathrm{C}$ ionization. The only surface reactions incorporated are $\mathrm{H}_{2}$ formation
Table 2. Model parameters for the various disk models. The columns denote the inner and outer radius in $\mathrm{AU}$, the gas mass in $M_{\oplus}$, the dustto-gas mass ratio $\delta$, the dust absorption cross section in the $\mathrm{UV}$ in $\mathrm{cm}^{2}$ per $\mathrm{H}$-atom and the type of UV radiation field. In the last colum, " $\mathrm{CH}$ " denotes a pure chromospheric UV radiation field, while "IS" stands for an interstellar Habing radiation field with $G_{0}=1$.

\begin{tabular}{lrlllc}
\hline \hline Name & $R_{\mathrm{i}}$ & $M_{\text {gas }}$ & $\delta$ & $\sigma$ & $F_{v}$ \\
\hline m0.033_190_CH & 190 & 0.033 & 1.73 & $3.37(-21)$ & CH \\
m0.33_190_CH & 190 & 0.33 & $1.73(-1)$ & $3.37(-22)$ & CH \\
m3.3_190_CH & 190 & 3.3 & $1.73(-2)$ & $3.37(-23)$ & CH \\
m0.033_190_IS & 190 & 0.033 & 1.73 & $3.37(-21)$ & IS \\
m0.33_190_IS & 190 & 0.33 & $1.73(-1)$ & $3.37(-22)$ & IS \\
m3.3_190_IS & 190 & 3.3 & $1.73(-2)$ & $3.37(-23)$ & IS \\
m0.033_60_CH & 60 & 0.033 & $2.3(1)$ & $5.38(-20)$ & CH \\
m3.3_60_CH & 60 & 3.3 & $2.3(-1)$ & $5.38(-22)$ & CH \\
m0.033_60_IS & 60 & 0.033 & $2.3(1)$ & $5.38(-20)$ & IS \\
m3.3_60_IS & 60 & 3.3 & $2.3(-1)$ & $5.38(-22)$ & IS \\
\hline
\end{tabular}

Note: numbers in parentheses: $3.37(-21)=3.37 \times 10^{-21}$.

and freezing out of $\mathrm{CO}$ on cold dust-grain surfaces. Since we are dealing with large dust particles, we reduced the $\mathrm{H}_{2}$ formation rate according to the reduced grain surface area. The abundance of $\mathrm{CO}$ ice is due to a balance between freezing out of gaseous $\mathrm{CO}$ and re-evaporation of $\mathrm{CO}$ ice. The two differences to the Kamp \& Bertoldi (2000) paper are: (1) the inclusion of cosmic ray reactions and (2) a lower temperature of $20 \mathrm{~K}$ for freezing out of $\mathrm{CO}$ ice. However, because of the strong stellar UV radiation field, the chemistry is mostly driven by stellar photons. A modified Newton-Raphson algorithm is used to obtain a stationary solution of the entire chemical network.

\section{Results}

Four main questions are adressed in the following subsections:

- What is the chemical composition of the disk? How does it depend on disk mass, what is the role of shielding?

- How does the CO-to- $\mathrm{H}_{2}$ ratio change with disk mass?

- How does the varying dust-to-gas mass ratio affect the chemical composition of the disk?

- What is the effect of a chromosphere as compared to the interstellar radiation field? When does a chromosphere need to be included in disk modeling?

The basic parameters of the disk models computed to answer these questions are summarized in Table 2 . The outer radius is fixed to $470 \mathrm{AU}$ in all models. The name of the disk model is composed as follows: $M_{\text {gas } \_} R_{\mathrm{i}_{-}} F_{\nu}$. The type of radiation field $F_{v}$ is indicated by "CH" or "IS", which stands for "chromosphere" or "interstellar". The former radiation field is explained in great detail in Sect. 2.2. The interstellar radiation field is taken from Habing (1968) and approximated by the following expression

$$
\begin{aligned}
& F_{v}=\frac{10^{-14}}{f_{5}^{2}}\left(-\frac{25}{6} f_{5}^{-2}+\frac{25}{2} f_{5}^{-1}-\frac{13}{3}\right) \\
& \operatorname{erg~cm}{ }^{-2} \mathrm{~s}^{-1} \mathrm{~Hz}^{-1} \text {, }
\end{aligned}
$$



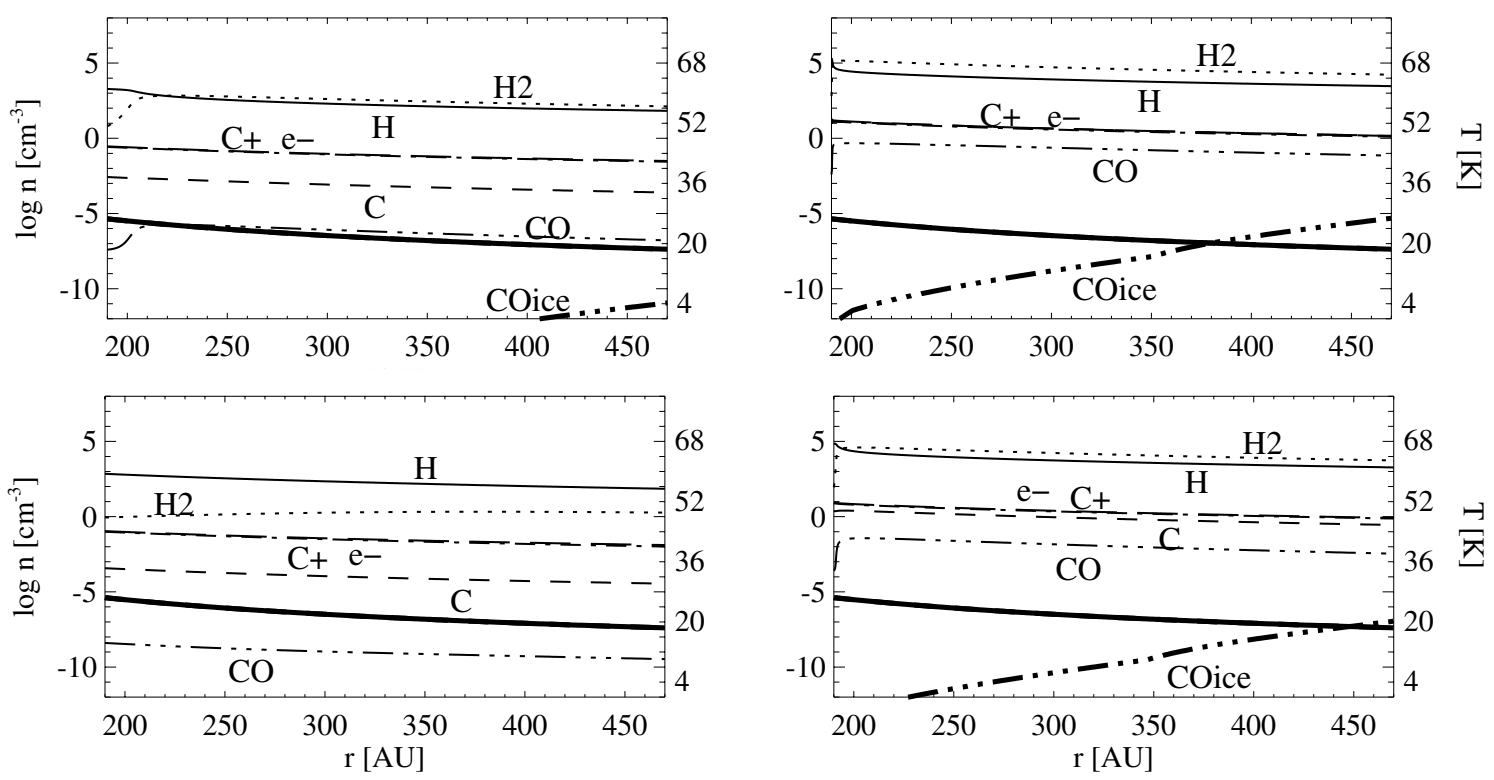

Fig. 4. Densities of different species (left scale) and temperature (right scale) in two different disk models: a) m0.033_190_CH model at the disk midplane; b) m3.3_190_CH model at the disk midplane; c) m0.033_190_CH model at one scaleheight; d) m3.3_190_CH model at one scaleheight. The adopted line styles are: $\mathrm{H}$ : solid, $\mathrm{H}_{2}$ : dotted, $\mathrm{C}$ : dashed, $\mathrm{C}^{+}$: dash-dotted, $\mathrm{CO}$ : dash-dot-dotted, $\mathrm{CO}_{\text {ice }}$ : dash-dot-dotted (thick), $\mathrm{e}^{-}$: long dashed, $T$ : solid (thick).

with $f_{5}$ being the wavenumber in $10^{5} \mathrm{~cm}^{-1}$. For simplicity, it is assumed that the interstellar radiation field only penetrates the disk from the inner edge. A more realistic approach would involve a $2 \mathrm{D}$ radiative transfer, which so far has not been included in the model.

\subsection{The chemical composition of the disk}

We limit the discussion here to some key species, namely $\mathrm{H}$, $\mathrm{H}_{2}, \mathrm{C}, \mathrm{C}^{+}, \mathrm{CO}$, and $\mathrm{CO}$ ice, that can act as possible tracers for the disk mass. The three models m0.033_190_CH, m0.33_190_CH, and m3.3_190_CH cover the transition from a completely transparent disk to a moderately shielded one.

Figure 4 illustrates the chemical structure of two disk models: m0.033_190_CH and m3.3_190_CH. Since the disks are optically thin in the UV, dust shielding is not important for the photoreactions. $\mathrm{H}_{2}$ can efficiently shield itself against photodissociation within one scaleheight in all three models. In the m3.3_190_CH model the molecular hydrogen even extends to 1.5 scaleheights. Despite this high abundance of molecular hydrogen, $\mathrm{H}_{2}$ is not able to shield $\mathrm{CO}$ against photodissociation. Self-shielding of CO sets in for the m0.33_190_CH disk model and becomes efficient in the highest mass model, m3.3_190_CH. There, the CO abundance reaches values of $\log \epsilon_{\mathrm{CO}}=-5.4$. In the outermost parts of the disk $r>390 \mathrm{AU}$, the temperature drops below $20 \mathrm{~K}$, but the low density $n_{\text {tot }}<$ $10^{5} \mathrm{~cm}^{-3}$ prevents a strong freeze-out of $\mathrm{CO}$ on dust grains.

The chemistry depends very much on the He abundance: helium is partially ionized by cosmic rays. The ionization degree $\mathrm{He} \mathrm{II/He} \mathrm{I} \mathrm{is} \mathrm{always} \mathrm{much} \mathrm{smaller} \mathrm{than} 1$ and depends strongly on the recombination processes and hence on the densities in the disk model. Since we use here the solar He abundance of $\log \epsilon_{\mathrm{He}}=0.1$, the destructive reaction $\mathrm{He}^{+}+\mathrm{CO}$ preserves a high $\mathrm{C}^{+}$abundance throughout the disk in all models.
Table 3. $\left[\mathrm{CO} / \mathrm{H}_{2}\right]$ ratio for a sample of disk models.

\begin{tabular}{lr}
\hline \hline Name & {$[\mathrm{CO} / \mathrm{H} 2]$} \\
\hline m0.33_190_CH & $3.58 \times 10^{-7}$ \\
m3.3_190_CH & $1.12 \times 10^{-5}$ \\
m0.33_190_IS & $1.89 \times 10^{-6}$ \\
m3.3_190_IS & $6.22 \times 10^{-5}$ \\
m3.3_60_CH & $7.53 \times 10^{-5}$ \\
m3.3_60_IS & $3.65 \times 10^{-4}$ \\
\hline
\end{tabular}

\subsection{The CO-to- $\mathrm{H}_{2}$ ratio}

Figure 5 illustrates how the $\mathrm{CO}-$ to- $\mathrm{H}_{2}$ ratio changes throughout the disk for various disk models. With increasing disk mass, the $\mathrm{CO}$ abundance in the disk rises and hence the $\mathrm{CO}-$ to $-\mathrm{H}_{2}$ ratio increases. In general, the difference between a model with a chromospheric radiation field and one with an interstellar radiation field amounts at most to $\Delta \mathrm{CO}-$ to- $\mathrm{H}_{2}$ ratio $\sim 0.5$ dex.

For an interpretation of observations, which give rather an integrated picture of the $\mathrm{CO}$ in the disk, it is better to use the integrated $\left[\mathrm{CO} / \mathrm{H}_{2}\right]$ ratio of the various disk models (see Table 3)

$\left[\mathrm{CO} / \mathrm{H}_{2}\right]=\frac{\int \epsilon(\mathrm{CO}) \mathrm{d} V}{\int \epsilon\left(\mathrm{H}_{2}\right) \mathrm{d} V}$.

Given the tenuous character of these disks, an IS UV radiation field would penetrate the disk from all directions and therefore lead to a much higher $\left[\mathrm{H}_{2} / \mathrm{CO}\right]$ ratio even for the $3.3 M_{\oplus}$ model. Thus, we draw the tentative conclusion that the $\left[\mathrm{CO} / \mathrm{H}_{2}\right]$ conversion factor in these disks can be orders of magnitude smaller than the canonical value for molecular clouds, $\left[\mathrm{CO} / \mathrm{H}_{2}\right] \sim 10^{-4}$. These disks generally resemble the photon dominated regions (PDR) on the surfaces of molecular clouds, which are characterized by very high UV irradiation and densities ranging from $10^{3}$ to a few times $10^{5} \mathrm{~cm}^{-3}$. 

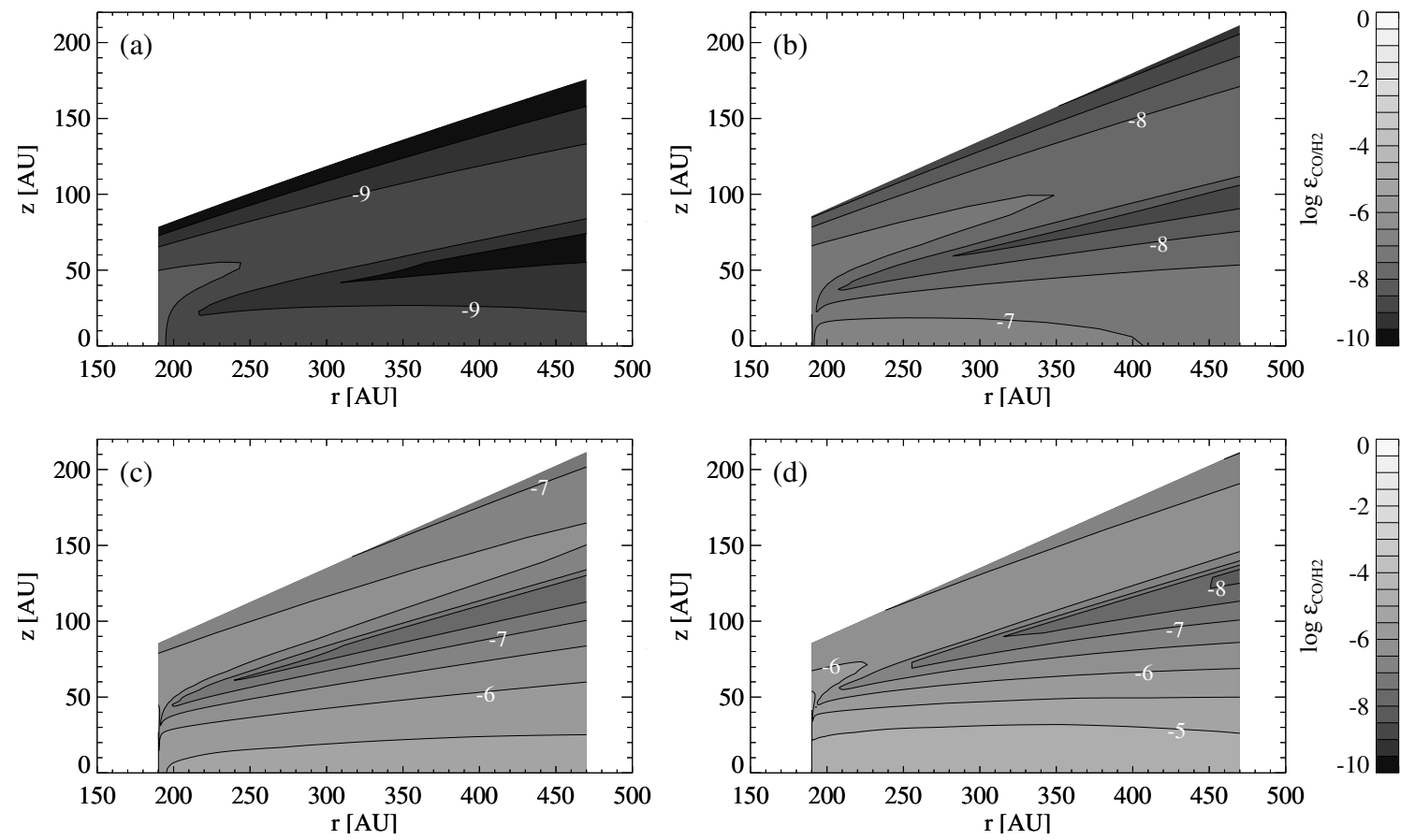

Fig. 5. The CO-to-H $\mathrm{H}_{2}$ ratio $\epsilon$ in 4 different disk models: a) m0.033_190_CH; b) m0.33_190_CH; c) m3.3_190_CH; and d) m3.3_190_IS.

\subsection{The role of dust}

As already stated above, dust shielding plays no role in the photochemistry in the disk. On the other hand, the dust grain surfaces are the location where molecular hydrogen is formed. Hence, the $\mathrm{H}_{2}$ formation rate

$R_{\mathrm{gr}}=3 \times 10^{-17}\left(\frac{T}{100 \mathrm{~K}}\right)^{1 / 2} \frac{\sigma_{\mathrm{uv}}}{6 \times 10^{-22} \mathrm{~cm}^{2}} \mathrm{~cm}^{3} \mathrm{~s}^{-1}$

is higher in models with a higher dust-to-gas mass ratio, e.g. m0.033_190_CH as compared to m3.3_190_CH. $\sigma_{\text {uv }}$ denotes here the effective $\mathrm{UV}$ attenuation cross section per $\mathrm{H}$ nucleus. A low dust-to-gas mass ratio of $1.73 \times 10^{-2}$ for the $0.033 M_{\oplus}$ model prevents formation of $\mathrm{H}_{2}$ throughout the disk.

The alternative model (Sylvester \& Skinner 1996) with an inner radius of $60 \mathrm{AU}$ and a dust mass of $0.77 M_{\oplus}$ has an even higher dust-to-gas mass ratio (m3.3_60_CH: 0.23 compared to m3.3_190_CH: $1.7310^{-2}$ ) and therefore $\mathrm{H}_{2}$ extends up to two scaleheights and $\mathrm{CO}$ up to one scaleheight in the disk. Additionally, the freezing out of $\mathrm{CO}$ is slightly enhanced, but still not efficient enough to obtain a high $\mathrm{CO}$ ice abundance. But even in these disk models with a high dust mass, dust absorption is not a dominant shielding process.

\subsection{The role of the chromosphere}

The outer parts of the disk, beyond $400 \mathrm{AU}$, are clearly dominated by the interstellar radiation field. Nevertheless, for the inner gas disk the chromospheric UV radiation is the driving mechanism in chemistry. If one neglects the presence of a chromosphere, the resulting $\mathrm{H}$ and $\mathrm{CO}$ abundances differ by more than a factor 10, especially in regions where most of the disk mass is located (see Fig. 6).
For somewhat younger stars, the chromosphere will be even more important, because the stronger stellar activity will give rise to an even higher UV photon flux. There will have been a point in time at which the disk was optically thick and actively accreting; therefore UV photons and X-rays from the accretion process have probably determined the chemistry and temperature balance in the upper layers of the protoplanetary disk. At which stellar age the transition to optically thin disks occurs as well as the point in time where accretion ceases and stellar activity takes over still has to be determined.

\section{Conclusion}

In this paper we investigate the chemical structure of tenuous circumstellar disks around young solar-type stars using the disk models developed by Kamp \& Bertoldi (2000). Previous observational studies have neglected the possible influence of a chromosphere for solar-type stars. Especially in an earlier evolutionary stage (WTTS), the UV radiation from the chromosphere is much stronger than that of the present Sun and may determine the chemistry in the circumstellar surrounding.

The main results of this work are:

- We present a recipe to calculate the chromospheric UV radiation field of young solar-type stars as a function of age.

- The CO-to- $\mathrm{H}_{2}$ ratio in low mass disks around solar-type stars is smaller than the canonic value of $10^{-4}$ for molecular clouds and resembles more closely the values found for the PDR surface layers of molecular clouds.

- The dust-to-gas mass ratio has a strong influence on the molecular hydrogen abundance in disks. Hydrogen, which would normally be atomic in low-mass disk, can become molecular in the presence of a high dust-to-gas mass ratio. This is simply because of an enhanced $\mathrm{H}_{2}$-formation rate 

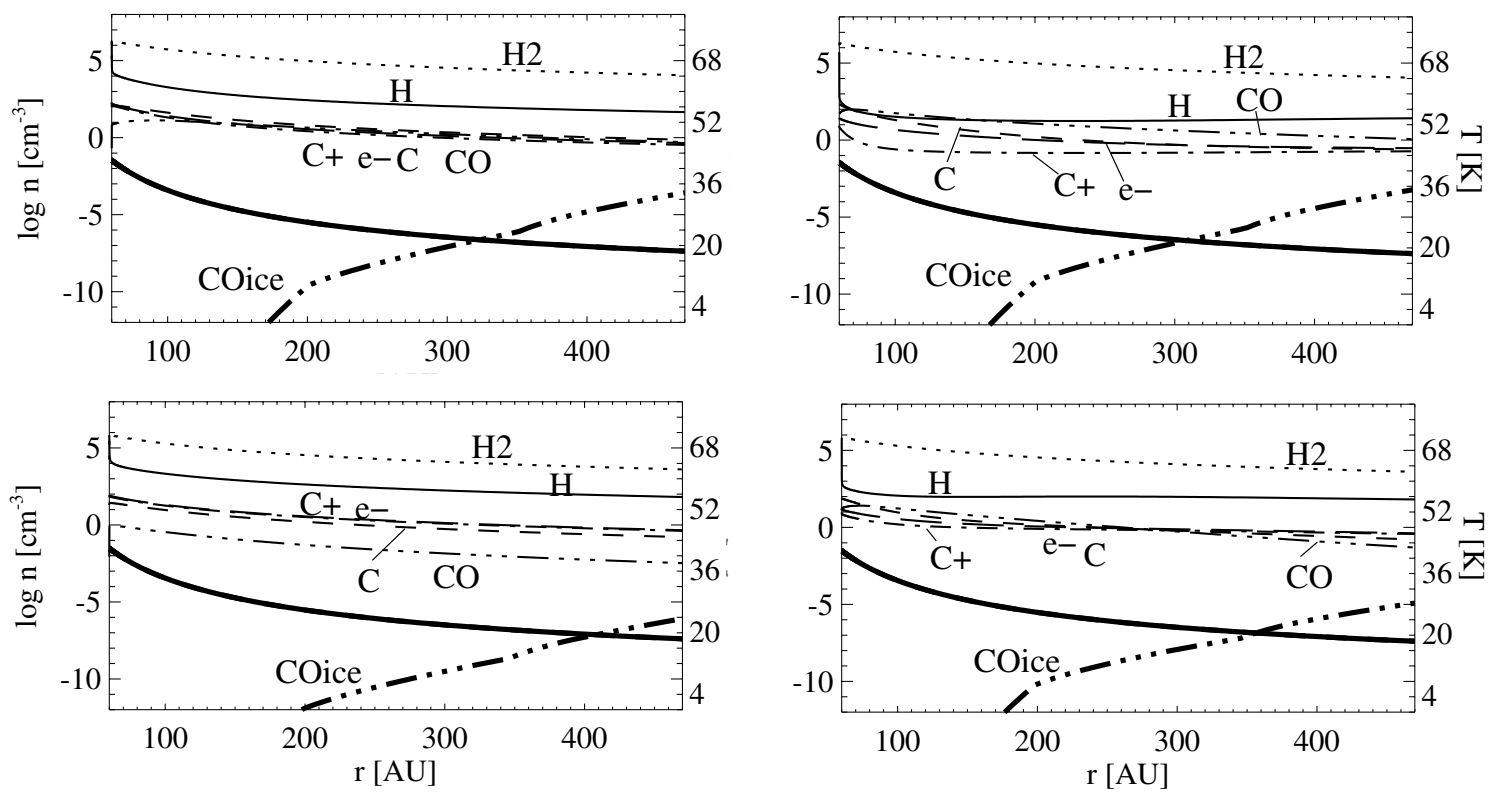

Fig. 6. Densities of different species (left scale) and temperature (right scale) in two different disk models: a) m3.3_60_CH model at the disk midplane; b) m3.3_60_IS model at the disk midplane; c) m3.3_60_CH model at one scaleheight; d) m3.3_60_IS model at one scaleheight. The adopted linestyles are: $\mathrm{H}$ : solid, $\mathrm{H}_{2}$ : dotted, $\mathrm{C}$ : dashed, $\mathrm{C}^{+}$: dash-dotted, $\mathrm{CO}$ : dash-dot-dotted, $\mathrm{CO}_{\text {ice }}$ : dash-dot-dotted (thick), $\mathrm{e}^{-}$: long dashed, $T$ : solid (thick).

and not because of dust shielding. The disks are optically thin in the UV continuum.

- The chromosphere of a 70 Myr old star dissociates the CO in the inner disk $(r<400 \mathrm{AU})$ of a solar-type star. Due to self-shielding effects, $\mathrm{H}_{2}$ is much less affected than CO. In the outer disk, the stellar UV radiation field is smaller than the typical interstellar UV field.

This work presents a first step towards the modeling of protoplanetary disks around young solar-type stars. The model is limited by the fact that we did not include an interstellar UV radiation field in a self-consistent way (2D radiative transfer). Moreover, at least simple line radiative transfer should be included to make it possible to (a) calculate the gas temperature in these disks self-consistently and (b) extend these disk models to higher masses.

Acknowledgements. The authors thank Ewine van Dishoeck for many fruitful discussions during the course of this work. We are grateful to the referee, whose comments helped to make this paper clearer and more concise and to Jeff Valenti for a careful reading of the manuscript. Astrochemistry in Leiden is supported by a SPINOZA grant from the Netherlands Organization for Scientific Research (NWO).

\section{References}

Ayres, T. R. 1997, JGR Planets, 102, 1641

Coulson, I. M., Walther, D. M., \& Dent, W. R. F. 1998, MNRAS, 296, 934

Dorren, J. D., \& Guinan, E. F. 1994, ApJ, 428, 805

Dunkin, S. K., Barlow, M. J., \& Ryan, S. G. 1997, MNRAS, 286, 604

Dunkin, S. K., Barlow, M. J., \& Ryan, S. G. 1997, MNRAS, 290, 165

Greaves, J. S., Coulson, I. M., \& Holland, W. S. 2000, MNRAS, 312, L1

Habing, H. J. 1968, Bull. Astr. Inst. Netherlands, 19, 421

Jura, M., \& Kahane, C. 1999, ApJ, 521, 302

Kalas, P., Graham, J. R., Beckwith, S. V. W., Jewitt, D. C., \& Lloyd, J. P. 2002, ApJ, 567, 999

Kamp, I., \& Bertoldi, F. 2000, A\&A, 353, 276

Kurucz, R. L. 1992, Rev. Mex. Astron. Astrofis., 23, 181

Mora, A., Merín, B., Solano, E., et al. 2001, A\&A, 378, 116

Scoville, N. Z., Sargent, A. I., Sanders, D. B., et al. 1986, ApJ, 303, 416

Sylvester, R. J., \& Skinner, C. J. 1996, MNRAS, 283, 457

Sylvester, R. J., Skinner, C. J., Barlow, M. J., \& Mannings, V. 1996, MNRAS, 279, 915

Zuckerman, B. 2001, ARA\&A, 39, 549

Zuckerman, B., Forveille, T., \& Kastner, J. H. 1995, Nature, 373, 494 